\title{
Site-Selected Transposon Mutagenesis at the hcf106 Locus in Maize
}

\author{
Lekha Das and Robert Martienssen ${ }^{1}$ \\ Cold Spring Harbor Laboratory, Box 100, Cold Spring Harbor, New York 11724
}

\begin{abstract}
The High chlorophyll fluorescence106 (Hcf106) gene in maize is required for chloroplast membrane biogenesis, and the hcf106-mum1 allele is caused by the insertion of a Robertson's Mutator Mu1 element into the promoter of the gene. SeedIings homozygous for hcf106-mum1 are pale green and die 3 weeks after germination, but only in the presence of Mutator activity conferred by active, autonomous $M u$ regulatory transposons elsewhere in the genome. When $M u t a t o r$ activity is lost, the mutant phenotype is suppressed, and homozygous plants have an almost wild-type phenotype. To isolate derivative alteles at the hcf106 locus that no longer require Mutator activity for phenotypic expression, we have developed a method for site-selected transposon mutagenesis in maize. This procedure, first described for Caonorhabditis elegans and Drosophila, involves using polymerase chain reaction (PCR) to screen pools of individuals for insertions and deletions in genes of known sequence. Pools of seedlings segregating for the progenitor allele hcf106-mum1 were screened by PCR for insertions and deletions associated with Robertson's Mutator. In a 360-bp target region, two new insertions and one deletion were identified in only $700 \mathrm{Mu}$-active gametes screened. One of the insertions was in the progenitor hcf106-mum1 allele and the other was in the wild-type allele, but all three new alleles were found to have breakpoints at the same nucleotide in the first intron. Unlike the hof106-mum1 progenitor allele, the deletion and one of the insertions conferred pale green seedling lethal phenotypes in the absence of Mutator activity. However, the second insertion had a weak, viable phenotype under these conditions. Although the sample size was small, our results suggest that this procedure can be used to rapidly identify transposon insertions into known genes in a single generation. Null derivative alleles can then be isolated in a second generation using the insertion as a starting point. This two-step procedure represents a powerful and simple way to "knock out" maize genes identified by sequence alone by using only a few thousand progeny from a simple cross.
\end{abstract}

\section{INTRODUCTION}

Transposable elements are versatile mutagens in microbes, plants, and animals because of their ability to disrupt genes by insertion and their ability to generate deletions, rearrangements, and point mutations once they are inserted at a given locus (McClintock, 1950; Berg and Howe, 1989). Most importantly, transpositions are typically conservative in transposon DNA sequences, providing disrupted target genes with molecular "tags." This property allows the cloning of genes defined only by the phenotypic consequences of transposon insertion, that is, by "transposon tagging" (Bingham and Judd, 1981; Fedoroff et al., 1984; Walbot, 1992). In principle, transposon tagging can be performed in reverse to identify individuals with transposon insertions in a gene that has already been cloned. These individuals can then be analyzed phenotypically to determine the function of the disrupted gene. This approach involves screening a large number of individuals for relatively rare insertion events.

The polymerase chain reaction (PCR) provides a sensitive means to identify transposon insertions via amplification of

\footnotetext{
1 To whom correspondence should be addressed.
}

transposon and target gene fusions. In Drosophila and Caenorhabditis elegans, pools of animals have been screened in this way to identify rare individuals that have "site-selected" transposon insertions in a given target gene (Ballinger and Benzer, 1989; Kaiser and Goodwin, 1990; Rushforth et al., 1993). Insertions can be identified by PCR amplification using primers from transposons and target genes. Unfortunately, these insertions often fail to result in a mutant phenotype because of the removal of the transposon from the primary transcript by splicing (Rushforth et al., 1993). However, by using PCR amplification, deletions, excisions, and rearrangements (derivative alleles) can be selected from pools of siblings that carry a transposon insertion at a given locus, resulting in a "gene knockout" (Zwaal et al., 1993). This two-step procedure (insertion followed by deletion) is particularly useful in the case of homozygous lethal mutations: it allows derivative alleles to be identified in viable $F_{1}$ heterozygous individuals. In contrast, phenotypic screens must utilize $F_{2}$ families segregating nonviable homozygotes. Furthermore, if the derivative phenotype is difficult to distinguish from the progenitor allele, phenotypic screens are impractical. Given the large number of gene 
sequences emerging from genome studies, site-selected reverse genetics represents a powerful way to identify the function of genes identified by sequencing alone.

In plants, numerous families of transposable elements are suitable for site-selected transposon mutagenesis. With few exceptions, these elements can mutate genes by both insertion and subsequent imprecise excision, deletion, and other rearrangements (Fedoroff, 1989; Walbot, 1992). In maize, Robertson's Mutator elements are particularly suited to this procedure. Robertson's Mutator elements fall into at least seven classes (reviewed by Chandler and Hardeman, 1992; Bennetzen et al., 1993). Functions required for transposition are supplied in trans by the Mutator regulatory element $M U D R$ (named in honor of D. Robertson), a 4.9-kb element with 200bp terminal inverted repeats that are shared with the other $M u$ element classes. $d M u D R$ elements are derived from MuDR elements by internal deletion (Chomet et al., 1991; Hershberger et al., 1991; Qin et al., 1991), but most of the other classes of $M u$ elements have unrelated internal sequences. Full-length MuDR elements encode two genes, at least one of which is required for transposition (Hershberger et al., 1991). A number of properties make $M u$ uniquely suited for site-selected insertional mutagenesis. First, $M u$ elements can accumulate to very high copy numbers with very high forward transposition frequencies (Robertson, 1978, 1980). Second, Mu elements share long terminal inverted repeats that are very similar between different classes of element, providing sufficient sequence for multiple nested primers (Chandler and Hardeman, 1992). Third, germinal excision is rare, whereas transpositions and flanking deletions are very common: Mu1 elements at the Alcohol dehydrogenase1 and Bronze2 loci in maize are known to generate flanking deletions resulting in null alleles at frequencies approaching 1\% (Taylor and Walbot, 1985; Levy and Walbot, 1991). Such deletions are typically less than $100 \mathrm{bp}$ and are thought to result from either abortive transposition or illegitimate recombination between the element and the gene. In the latter case, part of the element is lost in the deletion (Levy and Walbot, 1991).

We have used Robertson's Mutator elements to generate insertions and flanking deletions at the high chlorophyll fluorescence106 (hcf106) locus in maize by using site-selected transposon mutagenesis. The Hcf106 gene is required for chloroplast membrane biogenesis (Barkan et al., 1986), and the hcf106-mum1 allele results from the insertion of a Mu1 element in the hcf106 promoter (Martienssen et al., 1989; Barkan and Martienssen, 1991). hct106-mum1 was the first Mutator-induced mutation in maize to be shown to be suppressible. That is to say, the mutant phenotype is suppressed, and homozygous plants are phenotypically wild type (or nearly so) when Mutator activity is lost (Martienssen et al., 1990). When Mutator activity is present (in "Mu-active plants"), homozygous seedlings are pale green and nonphotosynthetic, and they die 3 weeks after germination when their seed reserves are exhausted. A number of suppressible mutations at other maize loci have subsequently been described, and those loci that have been cloned all have a $\mathrm{Mu}$ element inserted in the promoter region of the suppressible allele (reviewed in Martienssen and Baron, 1994). In the case of hct106, a Mu1 element is inserted at the third of three transcription initiation sites (Barkan and Martienssen, 1991). In Mu-active plants, no transcripts from the locus accumulate to significant levels. However, when Mutator activity is lost, chimeric transcripts arise at the junction of the Mu1 element and the Hcf106 gene. These transcripts provide sufficient $\mathrm{Hcf106}$ gene product to rescue the mutant phenotype, resulting in viable, fertile homozygous plants that have near-normal chlorophyll pigmentation.

Homozygous mutant seedlings have occasional dark green sectors resulting from excision of the Mut element from the Hcf106 gene (Martienssen et al., 1989). They can also have larger dark green sectors resulting from somatic loss of Mutator activity, which is correlated with methylation of Mu1 and MUDR elements in these sectors (Martienssen et al., 1990; Martienssen and Baron, 1994). Because of the frequent loss of Mutator activity in maize stocks carrying the original allele of hcf106 (Martienssen and Baron, 1994), it has proven to be very difficult to perform genetic tests (e.g., double mutant studies) using this allele because phenotypic suppression greatly confuses the results. Additional biochemical analysis of the chloroplast proteins affected by the hof106-mum1 mutation suggests that it may be leaky: residual levels of the affected thylakoid membrane proteins accumulate in homozygous mutant plants (Barkan et al., 1986). This leakiness could be due to low levels of transcript accumulation from the mutant allele (Barkan and Martienssen, 1991). In contrast to this suppressible allele, deletions that encompass part of the coding region would not be expected to result in a functional gene product regardless of transcription from the locus. For this reason, we were interested in isolating deletion derivatives at the hcf106 locus.

We have used PCR-based site-selected mutagenesis to isolate three new alleles at the Hcf106 locus by transposon insertion and deletion. Two of these alleles behave genetically as loss-of-function derivatives, and the third has an almost wildtype phenotype. As in studies with Drosophila and C. elegans, this provides a potentially powerful method for identifying the function of cloned genes via gene knockouts and allelic series.

\section{RESULTS}

Figure 1A shows a map of the hcf106-mum1 allele, based on cDNA and genomic sequencing (A. Baron and R. Martienssen, unpublished results). To generate null derivative alleles at the hcf106 locus, we developed a strategy to screen for deletions that flanked the Mu1 element in hcf106-mum1 and encompassed the initiator ATG codon in the first exon of the gene. DNA was purified from pools of heterozygous hof106-mum1/+ and $+l+$ seedlings and digested with Taql, which has a unique site that overlaps the first in-frame ATG codon (Figure 1). DNA from each pool was then amplified with primers from the $M u 1$ element and from the second exon of the gene, as described 


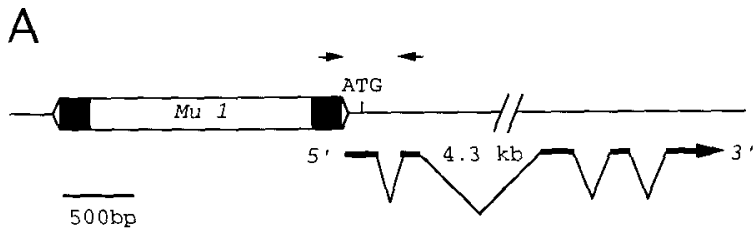

B

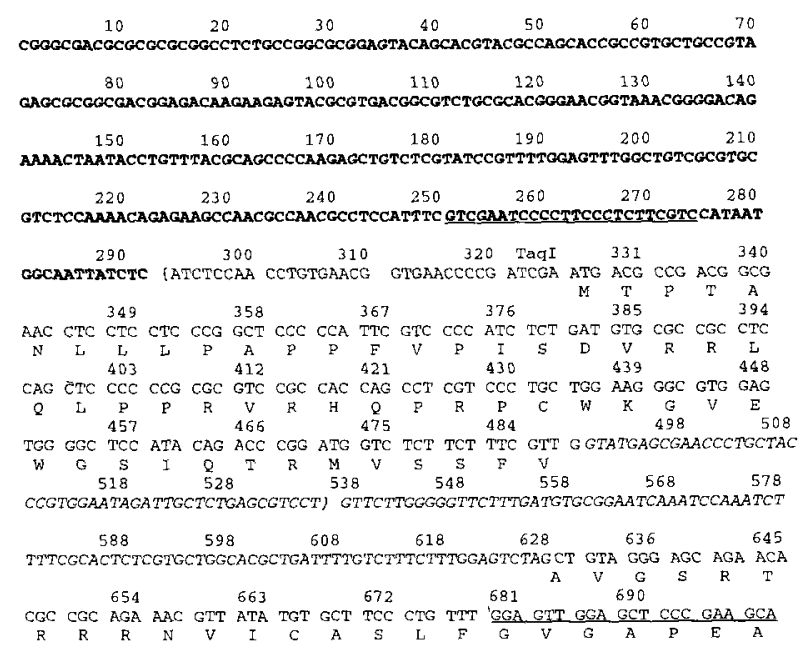

Figure 1. The Mur Insertion Site at the hof106 Locus.

(A) A map of the hcf106-mum1 allele is shown. The Mut element is inserted at the third of three transcript initiation sites (Barkan and Martienssen, 1991). The location of the initiator ATG and the primers described in the text (arrows) are shown. The second intron of $4.3 \mathrm{~kb}$ is shown, but not to scale (broken line).

(B) The sequence of part of the region shown in (A) from the end of the Mut element to the second exon of the Hcf106 gene is shown. The sequence of the Mut element is in boldface letters, and the intron sequence is in italics. Sequences corresponding and complementary to the primers used to screen seedling pools are underlined (see Methods). The 244-bp region deleted in hcf106-mum3 is indicated by parentheses (position 293 to 536 ).

below. Digestion with Taql prevents amplification of DNA from the progenitor hof106-mum1 allele because the primers are separated by the restriction site. Only undigested DNA that had lost the Taql site could be amplified so that deletion derivatives could be identified among the pools of seedlings. These derivatives were expected to give amplification products of 300 bp or less, whereas residual undigested DNA from the progenitor allele was expected to give a 400-bp amplification product.

Flanking deletions that retain the original insertion are thought to arise via abortive transpositions (Levy and Walbot, 1991) so that Mutator activity would be required to generate deletions in hcf106/+ parent plants. However, Mutator activity is readily lost from the parental stocks carrying hof106-mum1 (Martienssen and Baron, 1994). The pooled seedlings were therefore generated by crosses between $\mathrm{Mu}$-active hcf106/+ and $M u$-inactive wild-type $+/+$ plants. Mutator activity was monitored in the crosses by including the dominant Les28 (Lesion-mimic) marker in the Mu-inactive $+1+$ pollen parents. The Les28 mutation requires Mutator activity for phenotypic expression (Martienssen and Baron, 1994). Furthermore, crosses between $M u$-active and $M u$-inactive lines typically result in Mu-active progeny (Martienssen et al., 1990; Brown and Sundaresan, 1992). Thus, only Mu-active hcf106/+ parental plants produced progeny segregating for the lesion-mimic phenotype, which was otherwise suppressed in the Mu-inactive pollen parents. The Les 28 mutation is useful in this regard because Mutator activity can be monitored in the seedling (and therefore in the embryo) rather than in the aleurone layer (a derivative of the endosperm) so that heritable changes in $\mathrm{Mu}$ tator activity can be recorded (Martienssen and Baron, 1994). However, in cases where Mutator activity is retained at high levels, it would be more convenient to use mutable anthocyanin markers expressed in the aleurone layer (Walbot, 1986; Brown and Sundaresan, 1992).

Fifteen hundred seeds were planted from six crosses that resulted in lesion-mimic progeny, but only approximately onequarter of the resulting seedlings displayed the lesion-mimic phenotype. The $\mathrm{Mu}$-inactive parents of these crosses were heterozygous for Les 28 , indicating that a $50 \%$ loss of activity had most likely occurred in the germ line of the hcf106-mum1/+ parents (Martienssen and Baron, 1994), so that only 700 to $800 \mathrm{Mu}$-active gametes were represented by these seedlings. Seedlings were planted in three grids comprising 16 rows and 16 columns, with each position in the grid occupied by two seedlings ( 512 seedlings per grid), as shown in Figure 2. DNA was prepared from rows and columns by pooling $50 \mathrm{mg}$ of leaf tissue from seedlings in each row and separately from an additional $50 \mathrm{mg}$ of leaf tissue from seedlings in each column (32 DNA samples per grid). The pool size was chosen to avoid amplifying DNA from somatic sectors that were not germinally transmitted. We observed pale green somatic sectors on most heterozygous plants, and at least some of these resulted from transposition of $\mathrm{Mu}$ elements into the Hcf106 gene during leaf development (R. Martienssen and A. Baron, unpublished data). These sectors are typically small (one-sixteenth of a leaf or less), but if the pool size were too large, more amplification cycles would have been required, and products corresponding to pooled somatic sectors might have confused the analysis.

DNA was amplified from each pool by PCR using a primer complementary to the DNA sequence $50 \mathrm{bp}$ from the end of the Mu1 element and a primer from the second exon of the Hcf106 gene (Figure 1B). After we adjusted the conditions to reduce the abundance of background bands (see Methods), one position that resulted in a 200- to 300-bp PCR product was identified in each of the three grids by cross-referencing rows and columns. An example of one such position is shown in Figure 2. DNA was prepared from each of the plants at these positions, and the PCR products were amplified individually. Three candidate plants were identified, and PCR products from each were reamplified using a seminested primer complementary to the first $17 \mathrm{bp}$ of the Mu1 element; this sequence is 


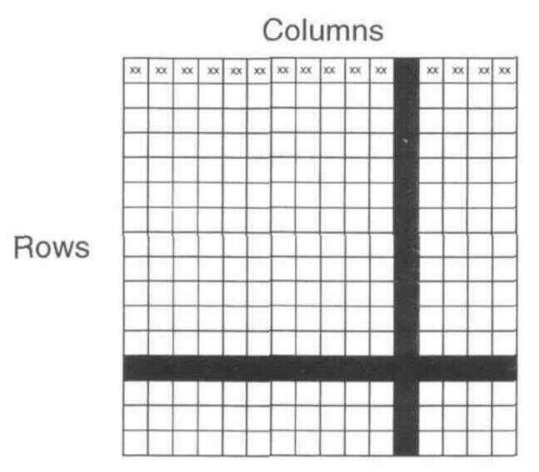

Rows

Columns
M 123345678

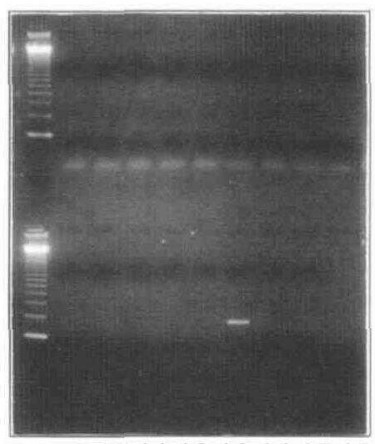

910111213141516
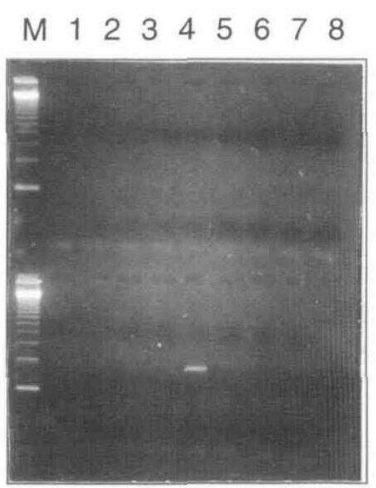

910111213141516
Figure 2. PCR Reactions with Pooled Samples.

Seed were germinated in a $16 \times 16$ grid, with two seedlings at each position in the grid as shown (x). DNA was extracted from pools of 32 seedlings from each row and each column and amplified using primers from the Mu1 element and the second exon of the gene as described in the text. Each lane of each gel was loaded with PCR products from individual rows and columns. $M$ indicates molecular weight marker lanes. The same derivative allele was amplified in one row and one column and was thus identified by a unique address.

known to be nearly identical in all known $M u$ elements. This primer also contained a restriction site for ease of cloning. The reamplified products were cloned and sequenced (see Methods). In each case, the sequence flanking the Mut element corresponded to a breakpoint located in the first intron of the gene; this breakpoint is 244 bp downstream of the Mu1 insertion site in the progenitor allele (Figure 1B). No other changes were observed.

The three plants resulted from three different crosses, indicating that they must represent independent derivative alleles. To confirm independence, DNA gel blotting was performed on DNA from each of the three plants using single and double digests. The blots were hybridized using a probe that encompassed the first and second exons and the first intron of the Hcf106 gene (p106K; see Methods). An example of a DNA gel blot is shown in Figure 3, and a summary of the structure of the alleles is shown in Figure 4. Only one of the derivatives, hcf106-mum3, corresponded to a simple 244-bp deletion of the type envisaged by the selection scheme (Figure 4). The other two had restriction maps consistent with the insertion of new Mu elements at the same location in the second intron of the progenitor hcf106-mumt allele and in the second intron of the wild-type allele, respectively (Figure 4). Insertions were recovered because new insertions of $\mathrm{Mu}$ elements between the Taql site and the second exon also allow amplification with the primers being used. However, the high frequency of these insertion events was unexpected (two new insertions in 700 Mu-active gametes).

By restriction mapping, PCR, and DNA gel blot analysis, the insertions in hcf106-mum2 and hcf106-mum 4 were found to be Mu1 and $d M u D R$ elements, respectively. In the case of hct106-mum2, digestion of genomic DNA with Sstl, Hindlll, and BamHI was used to show that an additional 1.4-kb insertion was present at the hcf106-mum1 locus. Digestion with Notl revealed that this insertion had a Notl site oriented opposite to that in hcf106-mum1 (Martienssen et al., 1989). The only Mu elements that have Notl sites but no Sstl, HindIII, or BamHI sites are Mu1 elements. PCR analysis confirmed that the original Mu1 element was still present at the locus (see Methods). In the case of hcf106-mum4, digestion with the same enzymes indicated a 2.3-kb insertion containing two Hindlll sites and two partially digested Sstl sites. Reprobing of the same DNA gel blot indicated that the BamHI fragment (Figure 3) and the partial Sstl fragments (data not shown) hybridized with a probe from the central portion of the MuDR element. This suggested that the element inserted in the hcf106-mum4 allele might be a deletion derivative of the 4.9-kb MuDR element. This was

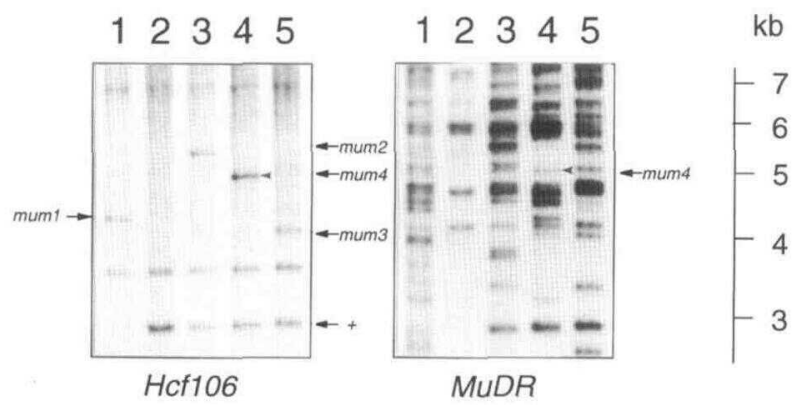

Figure 3. DNA Gel Blot Analysis of Derivative Alleles.

DNA samples from plants homozygous for hcf106-mum1 (lanes 1) or wild-type Hcf106 (inbred B73, lanes 2) and plants heterozygous for hcf106-mum2 (lanes 3), hcf106-mum4 (lanes 4), and hcf106-mum3 (lanes 5) were digested with $\mathrm{BamHI}$ and subjected to DNA gel blot analysis using hybridization probes from the first two exons of the Hcf106 locus or from the MuDR element. A hybridizing DNA fragment specific for each allele is marked with an arrow on the left panel. The hcf106-rmum4 allele is marked with an arrow on the right panel and with arrowheads in lanes 4. (+) marks the wild-type (B73) allele. Molecular length markers are given at right. 


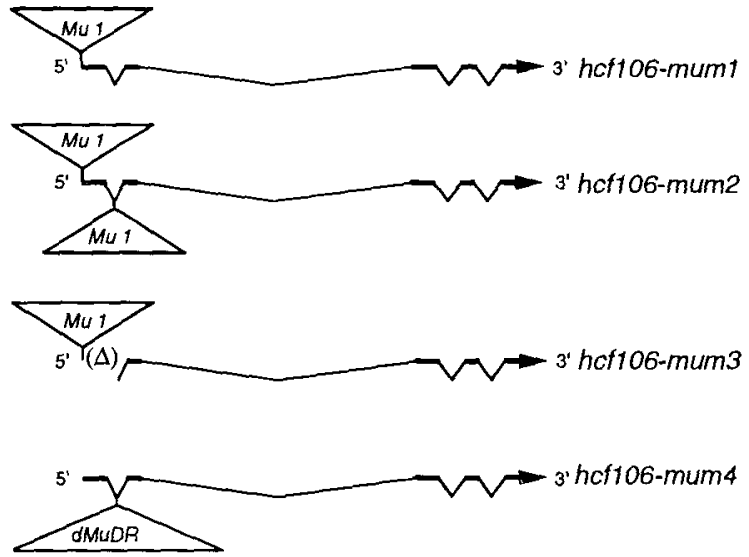

Figure 4. Schematic Maps of Progenitor hcf106-mumt and Derivative hof106-mum2, hcf106-mum3, and hcf106-mum4 Alleles.

Exon sequences are represented by horizontal lines; introns are shown as dips. The deletion in hcf106-mum3 is shown as a triangle.

confirmed by amplification with a primer (5'-TAAGGAGCTCCAAGCGCTGTCACC-3) specific to the end of the MUDR element (Hershberger et al., 1991) and an Hcf106 primer (5'-CCAGTGCTTCGGGAGCTCCAACTC-3). A 290-bp fragment was obtained confirming that the insertion was related to $M U D R$ (data not shown). Digestion with HindIII alone confirmed that the MUDR insertion was in the wild-type (B73) allele in the case of hof106mum4. This is because a polymorphic Hindlll site $4.5 \mathrm{~kb}$ upstream of the Hcf106 gene in B73 is absent in hcf106-mum1, which has a Hindlll site $10 \mathrm{~kb}$ upstream. In hcf106-mum4, HindIII fragments of 6 and $1.5 \mathrm{~kb}$ were observed; they could only have arisen if the progenitor allele in this case was the B73 allele (data not shown). Amplification using a primer upstream of the Mu1 insertion site in hcf106-mum1 (see Methods) and a primer from $M u$ gave a 300-bp product, confirming that there was no Mu1 element at the hof106-mum1 insertion site in the hof106mum4 allele.

Phenotypes conferred by each allele were determined by self-pollinating the heterozygous plants and planting the progeny in growth chambers. All three families segregated for unrelated white and yellow seedling lethal phenotypes found in the parent line and typical of Mutator lines. However, as expected, pale green lethal, high chlorophyll fluorescent hcf106-mum 2 and hcf106-mum3 seedlings appeared in a 1:3 ratio relative to normal green siblings in these families (see following data). PCR tests and DNA gel blotting of 20 individual and 20 pooled mutant and normal seedlings confirmed that the pale green seedlings were homozygous for the new allele (data not shown). Pale green lethal seedlings were not recovered from the hof106-mum4 families.

Plants homozygous for the progenitor hof106-mum1 allele are seedling lethal only when they contain active $M u$ elements (Martienssen et al., 1990). In the absence of Mutator activity, functional Hcf106 transcripts initiate within the Mu1 element at the locus so that the mutant phenotype is suppressed and homozygous plants are viable and of near-normal pigmentation (Barkan and Martienssen, 1991). Results from a typical self-pollination of $M u$-inactive hcf106-mum1/+ plants are shown in Table 1 (taken from Martienssen et al., 1990). Deletion derivative alleles would not be expected to be suppressed in this way because any $\mathrm{Mu}$-derived transcripts would lack the first exon of the gene. To investigate whether this was the case, progeny tests were performed using the new alleles, and $\mathrm{Mu}$ tator activity was monitored in the derivatives by using the Les 28 mutation, which confers a dominant lesion-mimic phenotype when Mu elements are active (Martienssen and Baron, 1994).

All three plants heterozygous for the original derivative alleles carried the Les 28 mutation but had lost the lesion-mimic phenotype by the seventh, fifth, and third leaves below the tassel, respectively (Martienssen and Baron, 1994). Following self-pollination and outcrossing, none of their progeny (of more than 200 examined) had the lesion-mimic phenotype. Further, when these progeny were self-pollinated, none of their progeny had the lesion-mimic phenotype either (of more than 300 examined). This suggested that these plants had lost $M u$ tator activity, and this supposition was confirmed by DNA gel blotting using methylation-sensitive enzymes and Mu1 probes. $M u$-active plants have unmethylated $M u 1$ elements, whereas Mu-inactive plants have mostly methylated elements (Chandler and Walbot, 1986; Bennetzen, 1987). Hinfl fragments from unmethylated Mu1-related elements migrate at 1.7 (Mu2) and 1.4 $\mathrm{kb}(M u 1)$, whereas methylated elements result in a ladder of fragments depending on methylation of Hinfl sites within and adjacent to the $M u$ element (Martienssen et al., 1990). Figure 5 shows that some of the original heterozygotes had predominantly unmethylated elements in the leaf from which DNA was extracted (arrows) but their progeny had predominantly methylated elements. This confirmed that their progeny had lost Mutator activity.

In progeny tests, plants heterozygous for hof106-mum 2 and plants heterozygous for hcf106-mum3 had nearly one in four mutant progeny when self-pollinated, even though they had lost Mutator activity (Table 1). In contrast, no mutant seedlings were observed when Mu-inactive hcf106-mum1 heterozygotes were self-pollinated (Table 1). Thus, hcf106-mum 2 and hcf106mum3 confer a novel Mu-independent phenotype, as predicted by the insertion and deletion identified in each allele by site-

Table 1. hcf106-mum2 and hct106-mum3 Confer a Novel, Mu-Independent Phenotype

\begin{tabular}{llll}
\hline $\begin{array}{l}\text { Parental } \\
\text { Genotypes }\end{array}$ & $\begin{array}{l}\text { Number } \\
\text { of } \\
\text { Progeny }\end{array}$ & $\begin{array}{l}\text { hof } \\
\text { Mutant } \\
\text { Seedlings }\end{array}$ & $\begin{array}{l}\text { Mutator } \\
\text { Activity }\end{array}$ \\
\hline hcf106-mum1/+ selfed & 273 & 62 & Yes \\
hcf106-mum1/+ selfed & 293 & 0 & No \\
hcf106-mum2l + selfed & 158 & 32 & No \\
hof106-mum3I+ selfed & 293 & 67 & No \\
\hline
\end{tabular}




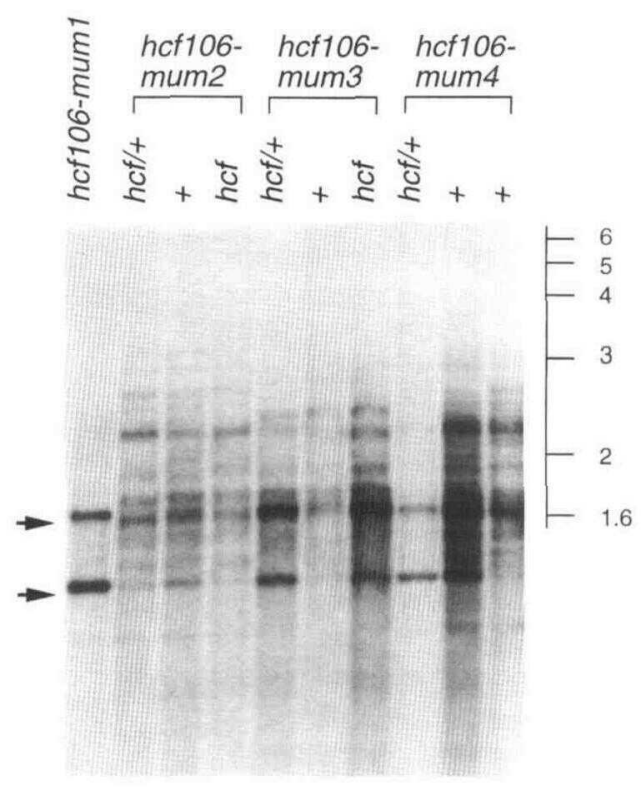

Figure 5. Methylation of Mu1 Elements in Progenitor and Derivative Families.

DNA samples were isolated from homozygous hcf106-mum1 seedlings from plants heterozygous for each derivative allele $(\mathrm{hcfl}+)$ and from their pooled normal $(+)$ and mutant (hcf) progeny following selfpollination. No hof progeny were recovered from hcf106-mum4. Samples were digested with Hinfl and analyzed by gel blot hybridization using the Mu1 element as a probe. Intensely hybridizing fragments at 1.4 and $1.7 \mathrm{~kb}$ (indicated by arrows) correspond to unmethylated Mu1 and Mu2 elements, respectively. Length markers at right are given in kilobases.

selected transposon mutagenesis. No pale green lethal seedlings appeared in hcf106-mum4 families, and DNA gel blot analysis revealed that this allele confers a subtly pale green viable phenotype when homozygous in $\mathrm{Mu}$-inactive backgrounds (data not shown). The effect of Mutator activity on the hcf106-mum4 allele is under investigation.

\section{DISCUSSION}

The derivative alleles of $h$ cf106 described here allow the $h$ cf106 null phenotype to be defined morphologically and biochemically. This is important because leaky expression from the progenitor hof106-mum1 allele might be responsible for the variable levels of some thylakoid proteins found in mutant seedlings (Barkan et al., 1986). We are now in a position to determine thylakoid structure and protein composition in chloroplast membranes from plants devoid of $\mathrm{Hcf} 106$ protein: the hcf106-mum3 allele in particular has completely lost the first exon of the gene that encodes the first two methionine codons. The new derivatives will also be useful in genetic experiments in which maintaining Mutator activity is impractical. This is because the phenotype conferred by the hcf106-mum2 and hcf106-mum3 derivatives no longer depends on Mutator activity, unlike the phenotype conferred by the progenitor allele hcf106-mum1 (Martienssen et al., 1990). When Mutator activity is lost, somatically or germinally, hcf106-mum1 expression is restored at the locus leading to accumulation of functional transcripts and suppression of the mutant phenotype (Barkan and Martienssen, 1991). Attempts to make double mutants and hypoploids have been greatly hampered by high-frequency loss of Mutator activity when the progenitor allele is introduced into non-Mutator lines (Martienssen and Baron, 1994). In contrast, hcf106-mum2 and hcf106-mum3 are mutant in the absence of Mutator activity, allowing multiple rounds of backcrosses into non-Mutator lines without loss of the mutant phenotype.

Site-selected transposon mutagenesis is a powerful method for identifying insertions and deletions in a target gene in populations of plants without phenotypic selection. In maize, Robertson's Mutator transposons are ideally suited for this approach because of their high copy number and conserved termini that allow a wide range of insertion alleles to be recovered using a single set of nested primers. Site-selected mutagenesis is particularly useful in the case of lethal mutations, such as hcf106, for which phenotypic screens are only possible in $F_{2}$ families. Another important application of this technique would be to isolate derivatives of dominant transposon-induced mutations. For example, at the Knotted locus in maize, the loss-of-function phenotype is not known, but there are several transposon-induced dominant alleles (Hake, 1992). By using PCR to select for derivative alleles as heterozygotes, deletions could be isolated from these dominant alleles without any prior knowledge of the mutant phenotype.

Among only $700 \mathrm{Mu}$-active gametes screened, two new insertions were identified at the hcf106 locus. One was found as a second insertion (hcf106-mum2) in the progenitor hcf106mum1 allele, and the other was found as a new insertion (hcf106-mum4) in the wild-type allele. Thus, new insertions in a known sequence can be recovered from a small population of plants in a simple one-step screen, even when the target sequence for new insertions is extremely small ( $\sim 360$ bp between the Taql site and the Hcf106 primer). The third allele, hcf106-mum 3 , was identified as a deletion derivative of the progenitor hcf106-mum1 allele from among the $350 \mathrm{Mu}$-active hcf106-mum1 gametes recovered (the other $350 \mathrm{Mu}$-active gametes were +). Thus, insertions and deletions were recovered at approximately equal frequencies (1 in 350). The small target size probably accounts for the fact that all three alleles had the same breakpoint in the gene. This breakpoint must represent a favored site for Mu insertion. Similarly, a preferred site was observed in two of three stable derivative alleles selected phenotypically at the Bronze2 locus in maize (Levy and Walbot, 1991), and similar numbers of deletion derivatives were recovered. Although the sample size is small, this suggests that other loci in maize are likely to exhibit a similar spectrum of insertions and deletions when selected in this way.

Two of the new hcf106 alleles behave genetically as loss-offunction derivatives (see previous discussion). Thus, the procedure we describe can be used to identify gene knockouts in maize, whereby insertions and deletions in a given gene 
can be obtained knowing only its DNA sequence. In principle, a handful of insertion alleles of a given gene can be obtained by screening a few thousand seedlings in grids using primers from the transposon and primers from the gene, as described here. Fifteen hundred seedlings (up to $3000 \mathrm{Mu}$ gametes depending on the cross) can be screened using only 96 DNA preparations and a single round of nested PCR.

To be able to use small numbers of seedlings is very useful when the genetic background is important for the screen. However, insertions are most efficiently generated using a large, systematic collection of transposon families, as has been done in C. elegans (Zwaal et al., 1993). In this system, frozen animals can be maintained for later recovery after identifying new insertion alleles in pooled DNA samples. A collection of maize seed has been developed for this purpose by Pioneer Hi-Bred (Johnston, IA) using $M u$ transposable elements, and $M u$ insertions into many target genes can be routinely obtained from this collection by site-selected PCR screening of the pooled parental DNA (S. Briggs, personal communication).

Unfortunately, as in studies with C. elegans and Drosophila, many insertions will not result in mutant phenotypes because they disrupt introns or noncoding flanking sequences or because they are removed from the primary transcripts by splicing within the element (Kaiser and Goodwin, 1990; Ortiz and Strommer, 1990; Rushforth et al., 1993). To overcome these limitations, deletion derivatives can be identified from insertion alleles by the method described here once insertions are recovered from the primary screen. These alleles can be used to define unequivocally the effects of deleting gene activity in the plant.

Transposon insertion has numerous advantages over transgenic approaches to gene knockouts, such as gene replacement or antisense inhibition. Gene replacement strategies are still highly inefficient and can only target one gene at a time. Furthermore, derivatives cannot be selected from the initial knockouts in most gene replacement strategies. Antisense transgenic plants have been widely used to reduce gene expression; however, antisense knockouts are dominant so that lethals are very difficult or impossible to observe. in contrast, site-selected transposon insertions can target an unlimited number of genes simply by using different primer combinations. Derivative alleles are easy to identify in the next generation, and last but not least, transposon knockouts are typically recessive so that lethal mutations can be readily recovered as viable heterozygotes. Thus, this technique represents a potentially powerful method for reverse genetics (gene knockouts) in plants, just as similar methods have been powerful in animals (Zwaal et al., 1993; Greenstein et al., 1994).

\section{METHODS}

The origins and maintenance of the high chlorophyll fluorescence106 (hch106) and Lesion-mimic (Les28) mutations were as described by Barkan et al. (1986) and Martienssen and Baron (1994). Mutator (Mu)active heterozygous $h c f 106 /+$ parents were identified by DNA gel blot analysis or by testcrosses and progeny screens. DNA preparation was by the urea-phenol extraction method of Cone (1989), and DNA gel blotting was as described by Martienssen and Baron (1994). The hybridization probes used were from the first two exons of the Hcf106 gene (p106K; Barkan and Martienssen, 1991) and the internal BamHIEcoRI fragment from the MuDR element (a gift from V. Chandler, University of Oregon, Eugene, OR). Pooled DNA samples were digested overnight with Taql, and polymerase chain reactions (PCRs) were performed using a primer from the end of $M u\left(5^{\prime}\right.$-TCGAATCCGCTTCTCTCTTCGTCC-3) and a primer from the second exon of the Hcf106 gene (5'-CCAGTGCTTCGGGAGCTCCAACTC-3). Cycling conditions are as follows: $1 \mathrm{~min}$ at $94^{\circ} \mathrm{C}, 1 \mathrm{~min}$ at $65^{\circ} \mathrm{C}$, and $2 \mathrm{~min}$ at $72^{\circ} \mathrm{C}$, for 35 cycles in a Perkin-Elmer Cetus Thermocycler. Ten microliters from each $50-\mu \mathrm{L}$ reaction were loaded on each lane of a mini-agarose gel containing $0.1 \mu \mathrm{g} / \mathrm{mL}$ ethidium bromide, and products were visualized with UV light after electrophoresis.

These conditions were established by "spiking" test reactions with dilutions of undigested DNA from the progenitor allele and determining conditions for which only spiked reactions gave a 400 -bp product. Background bands were observed with single primers and primer combinations when annealing temperatures were too low or when too many cycles were used. The presence of the same band in two different cross-referenced pools was used to confirm the authenticity of amplified products. Other primers from the end of MU1 (5'-CAGAATTCCATAATGGCAATTATCTC-3) and MUDR (5'-TAAGGAGCTCCAAGCGCTGTCACC-3) were also used to amplify specific alleles. PCR products were digested with EcoRI and Sstl and ligated into M13mp18 and M13mp19 for sequence analysis. DNA from the progenitor allele was amplified using primers from upstream (5'-GAATCAGACGGCTCAGGTTGTACC-3) and downstream (5'-CACATCAGAGATGGGGACGAATGG-3) of the Mu1 insertion site in combination with Mu1 primers under the same conditions.

\section{ACKNOWLEDGMENTS}

We thank Eric Richards for two-dimensional pooling suggestions, Tom Volpe and Derek Smith for help with DNA preparations, and Mark Settles for performing progeny tests. Thanks go to Marja Timmermans for commenting on the manuscript. This work was supported by grants from the Department of Energy (Grant No. DE-FG02-91ER20047) and the National Science Foundation (Grant No. MCB-9220774).

Received November 17, 1994; accepted January 11, 1995.

\section{REFERENCES}

Ballinger, D.G., and Benzer, S. (1989). Targeted gene mutations in Drosophila. Proc. Natl. Acad. Sci. USA 86, 9402-9406.

Barkan, A., and Martienssen, R.A. (1991). Inactivation of maize transposon $M u$ suppresses a mutant phenotype by activating an outward-reading promoter near the end of Mut. Proc. Natl. Acad. Sci. USA 88, 3502-3506.

Barkan, A., Miles, D., and Taylor, W.C. (1986). Chloroplast gene expression in nuclear, photosynthetic mutants of maize. EMBO J. 5 , 1421-1427. 
Bennetzen, J.L. (1987). Covalent DNA modification and the regulation of Mutator element transposition in maize. Mol. Gen. Genet. 208, 45-51.

Bennetzen, J.L., Springer, P.S., Cresse, A.D., and Hendrickx, M. (1993). Specificity and regulation of the Mutator transposable element system in maize. Crit. Rev. Plant Sci. 12, 57-95.

Berg, D.E., and Howe, M.M., eds (1989). Mobile DNA (Washington, DC: American Society for Microbiology).

Bingham, P.M., and Judd, B.H. (1981). A copy of the copia transposable element is very tightly linked to the $w^{\text {a }}$ allele at the white locus of $D$. melanogaster. Cell 25, 705-711.

Brown, J., and Sundaresan, V. (1992). Genetic study of the loss and restoration of Mutator transposon activity in maize: Evidence against dominant-negative regulator associated with loss of activity. Genetics 130, 889-898.

Chandler, V.L., and Hardeman, K. (1992). The Mu elements of Zea mays. Adv. Genet. 30, 77-122.

Chandler, V.L., and Walbot, V. (1986). DNA modification of a maize transposable element correlates with loss of activity. Proc. Natl. Acad. Sci. USA 83, 1767-1771.

Chomet, P.S., Lisch, D., Hardeman, K., Chandler, V.L., and Freeling, M. (1991). Identification of a regulatory transposon that controls the Mutator transposable element system in maize. Genetics 129 , 261-270.

Cone, K. (1989). Another maize DNA miniprep. Maize Genet. Coop. Newslett. 63, 68.

Fedoroff, N.V. (1989). Maize transposable elements. In Mobile DNA, D.E. Berg and M.M. Howe, eds (Washington, DC: American Society for Microbiology), pp. 375-411.

Fedoroff, N.V., Furtek, D.B., and Nelson, O.E., Jr. (1984). Cloning of the bronze locus in maize by a simple and generalizable procedure using the transposable element Activator (AC). Proc. Natl. Acad. Sci. USA 81, 3825-3829.

Greenstein, D., Hird, S., Plasterk, R.H.A., Andachi, Y., Kohara, Y., Wang, B., Finney, M., and Ruvkun, G. (1994). Targeted mutations in the Caenorhabditis elegans POU homeobox gene ceh-18 cause defects in oocyte cell cycle arrest, gonad migration and epidermal differentiation. Genes Dev. 8, 1935-1948.

Hake, S. (1992). Unravelling the knots in plant development. Trends Genet. 8, 109-114.

Hershberger, R.J., Warren, C.A., and Walbot, V. (1991). Mutator activity in maize correlates with the presence and expression of the Mu transposable element Mu9. Proc. Natl. Acad. Sci. USA 88, 10198.

Kaiser, K., and Goodwin, S.F. (1990). "Site-selected" transposon mutagenesis of Drosophila. Proc. Natl. Acad. Sci. USA 87, 1686-1690.
Levy, A.A., and Walbot, V. (1991). Molecular analysis of the loss of somatic instability in the bz2::mur allele of maize. Mol. Gen. Genet. 229, 147-151.

Martienssen, R.A., and Baron, A. (1994). Co-ordinate suppression of mutations caused by Robertson's Mutator transposons in maize. Genetics 136, 1157-1170.

Martienssen, R.A., Barkan, A., Freeling, M., and Taylor, W.C. (1989). Molecular cloning of a nuclear gene in maize involved in thylakoid organization and its regulation by Robertson's Mutator. EMBO J. 8 , 1633-1640.

Martienssen, R.A., Barkan, A., Taylor, W.C., and Freeling, M. (1990) Somatically heritable switches in DNA modification of $M u 1$ transposable elements in maize revealed by a suppressible mutant. Genes Dev. 4, 331-343.

Mcclintock, B. (1950). The origin and behavior of mutable loci in maize. Proc. Natl. Acad. Sci. USA 36, 344-355.

Ortiz, D.F., and Strommer, J.N. (1990). The Mu1 maize transposable element induces tissue-specific aberrant splicing and polyadenylation in two Adh1 mutants. Mol. Cell. Biol. 10, 2090-2095.

Qin, M., Robertson, D.S., and Ellingboe, A. (1991). Cloning of the Mutator transposable element MuA2, a putative regulator of somatic mutability of the a1-mum2 allele in maize. Genetics 129, 845-854.

Robertson, D.S. (1978). Characterization of a mutator system in maize. Mutat. Res. 51, 21-28.

Robertson, D.S. (1980). The timing of $M u$ activity in maize. Genetics 94, 969-978.

Rushforth, A.M., Saari, B., and Anderson, P. (1993). Site-selected insertion of the transposon TC1 into a Caenorhabditis elegans myosin light chain gene. Mol. Cell. Biol. 13, 902-910.

Taylor, L.P., and Walbot, V. (1985). A deletion adjacent to the maize transposable element Mur accompanies loss of $A d h 1$ expression. EMBO J. 4, 869-876.

Walbot, V. (1986). Inheritance of Mutator activity in Zea mays as assayed by somatic instability of the bz2-mu1 allele. Genetics 114, 1293-1312.

Walbot, V. (1992). Strategies for mutagenesis and gene cloning using transposon tagging and T-DNA insertional mutagenesis. Annu. Rev. Plant Physiol. Plant Mol. Biol. 43, 49-82.

Zwaal, R.R., Broeks, A., van Meurs, J., Groenen, J.T.M., and Plasterk, R.H.A. (1993). Target-selected gene inactivation in Caenorhabditis elegans by using a frozen transposon insertion mutant bank Proc. Natl. Acad. Sci. USA 90, 7431-7435. 
Site-selected transposon mutagenesis at the hef106 locus in maize.

L Das and R Martienssen

Plant Cell 1995;7;287-294

DOI 10.1105/tpc.7.3.287

This information is current as of December 13, 2013

Permissions
eTOCs

CiteTrack Alerts

Subscription Information
https://www.copyright.com/ccc/openurl.do?sid=pd_hw1532298X\&issn=1532298X\&WT.mc_id=pd_hw1532298 $\mathrm{X}$

Sign up for eTOCs at:

http://www.plantcell.org/cgi/alerts/ctmain

Sign up for CiteTrack Alerts at:

http://www.plantcell.org/cgi/alerts/ctmain

Subscription Information for The Plant Cell and Plant Physiology is available at: http://www.aspb.org/publications/subscriptions.cfm 